\title{
Covalent crosslinking of carbon nanostructures
}

\author{
URMIMALA MAITRA, M PANDEESWAR and T GOVINDARAJU* \\ New Chemistry Unit, Chemistry and Physics of Materials Unit, Jawaharlal Nehru Centre for Advanced \\ Scientific Research, Jakkur, Bangalore 560 064, India \\ e-mail: tgraju@jncasr.ac.in
}

MS received 29 September 2011; revised 16 December 2011; accepted 30 December 2011

\begin{abstract}
Covalent crosslinking of carbon nanostructures of different dimensionalities such as nanodiamond, single walled carbon nanotubes (SWNTs) and graphene can yield useful homo- and hetero-binary conjugates. Binary conjugation of the nanocarbons has been achieved by introducing symmetrical amide-linkages between acid (-COOH) functionalized nanocarbons and a diamine-linker. The binary conjugates have been characterized by using transmission electron microscopy as well as infrared, Raman and photoluminescence spectroscopies. Dispersions of covalently crosslinked binary conjugates of nanocarbons could be obtained in dimethyl formamide (DMF). Composites of the binary conjugates with polymer can be readily prepared by using the DMF suspensions.
\end{abstract}

Keywords. Covalent crosslinking; carbon nanostructures; binary conjugates; nanocomposites; electron microscopy; optical properties.

\section{Introduction}

One of the important factors that determines the nature and properties of nanomaterials is their dimensionality. Among the various nanomaterials synthesized and characterized in recent years, nanocarbons of different dimensionalities are of special interest. In the nanocarbon family, we have zero-dimensional nanodiamond, onedimensional carbon nanotubes and two-dimensional graphene. There has been a vast amount of research on the properties of the individual nanocarbons. Nanodiamond (ND) exhibits useful mechanical, electrical and optical properties. ${ }^{1-6}$ Beneficial properties of single walled carbon nanotube (SWNTs) are well-documented, ${ }^{7-9}$ while those of graphene $(\mathrm{G})$ have been discovered more recently. ${ }^{10}$ Binary combinations of nanocarbons with different dimensionalities may lead to integration of properties of the constituents units as well as result in new properties. It could be of some advantage if binary combinations of nanostructures are prepared by covalent modification over non-covalent methods. Covalently linked carbon nanostructures in particular, hetero-combinations will have better interactions among the two nanostructures which are easy to understand and may lead to improved properties. One of the effects of a component nanostructure in the conjugate may synergistically benefit the mechanical properties of the hetero-binary conjugate because of better mixing

*For correspondence which facilitates greater interactions among the individual components. It has been found recently that extraordinary synergy in mechanical properties resulted from incorporating two nanocarbons into polymer matrices. ${ }^{11}$ In this case the binary combination was achieved by just physical mixing. Covalently linked nanocarbons could provide better insight into the mechanism of origin of such synergistic effects reflected in the mechanical properties. Again ND-SWNT composites have been used to prepare cold cathodes. ${ }^{12}$ Covalently linked ND with SWNT can be studied for enhanced activities in such applications as well. In general, covalent conjugates of carbon nanostructures, in particular hetero-binary conjugates are fundamentally important in understanding the synergistic effect observed in the derived composites and may find applications as high performance materials. There are only few reports on the covalent crosslinking of carbon nanotubes. Chiu et $a l .{ }^{13}$ have reported crosslinking of two chloride terminated carbon nanotubes via diamines while Frehill et $a l .{ }^{14}$ have used inorganic metal complexes to crosslink carbon nanotubes. Bundles of carbon nanotubes can be linked by means of [2+1] cycloaddition using nitrenes. ${ }^{15}$ To our knowledge, there are no reports on the covalent conjugation of nanocarbons of different dimensionalities. We have therefore carried out covalent crosslinking of carbon nanostructures with similar or different dimensionalities. Besides the preparation of crosslinked NDs, SWNTs and G, we have synthesized different hetero-nanocarbon combinations such as 
ND + SWNT, ND + G and G + SWNT. Such homoand hetero-nanocarbon adducts may find use in special applications related to high performance materials.

\section{Experimental}

Nanodiamond (ND) with phase purity higher than $98 \%$ and an average particle size of around $5 \mathrm{~nm}$ was purchased from Tokyo Diamond Tools, Tokyo, Japan. Fewlayer graphene $(\mathrm{G})$ was prepared by the exfoliation of graphite oxide following the procedure described recently. ${ }^{16}$ Single walled carbon nanotubes (SWNTs) prepared by the DC arc-discharge process were treated with dilute $\mathrm{HCl}$ and heated under hydrogen atmosphere several times to remove the metal nanoparticles and amorphous carbon. ${ }^{17}$ Mono protected $N 1-(t$-Boc $)-1,2-$ ethylenediamine was synthesized following the modified procedure reported in the literature (Supporting Information). ${ }^{18}$

In order to prepare binary homoconjugates of ND, G and SWNT, each of these carbon nanostructures was treated to create surface carboxyl groups. A diamine linker was then used to covalently conjugate the acid functionalized nanocarbons. N,N'-Dicyclohexylcarbodiimide (DCC) was used as the coupling reagent to enhance the reaction of acid functionalized nanocarbons with the diamine. In a typical experiment with $\mathrm{ND}, 100 \mathrm{mg}$ of the nanocarbon was refluxed with conc. $\mathrm{HNO}_{3}(5 \mathrm{~mL})$ and conc. $\mathrm{H}_{2} \mathrm{SO}_{4}(45 \mathrm{~mL})$ for $12 \mathrm{~h}$. The acid-functionalized nanodiamond (ND-COOH) was washed with distilled water and dried under vacuum. ${ }^{19}$ SWNTs and G were functionalized with acid groups by heating a mixture of nanocarbon with conc. $\mathrm{HNO}_{3}$, conc. $\mathrm{H}_{2} \mathrm{SO}_{4}$ and water in a microwave oven for about 5-8 min under hydrothermal conditions, followed by further heating at $100^{\circ} \mathrm{C}$ for $6-8 \mathrm{~h}$ in an oven. The product thus obtained was washed with distilled water and filtered through a sintered glass funnel to yield acid functionalized nanocarbons SWNT-COOH and G-COOH. ${ }^{7,19-22}$

To prepare binary nanocarbon homoconjugates, the acid-functionalized nanocarbon $(5 \mathrm{mg})$ of interest was taken in DMF (20 mL), sonicated for 10 min for thorough mixing. To this mixture, coupling reagent DCC $(1 \mathrm{mg})$ and 1,2-ethylenediamine (ED) $(1 \mathrm{~mL})$ were added. Reaction mixture was stirred for $4 \mathrm{~h}$ under inert atmosphere and the products were collected by centrifugation. The product was washed with copious amounts of water, acetonitrile and ethanol. The sample was dried in vacuo and used for further characterization. For the preparation of binary heteroconjugates of nanocarbons, a two-step covalent crosslinking procedure was followed. Mono-protected Boc-1,2-ethylenediamine was first coupled to a acid functionalized nanocarbon through a amide linkage which was then subjected to bocdeprotection using trifluoroacetic acid. The nanocarbon with the free amine was coupled to another acid functionalized nanocarbon through an amide linkage to obtain the ED linked binary heteroconjugate. This procedure eliminates the possibility of obtaining undesired products resulting from inter- and intramolecular cross-coupling reactions. In a typical reaction an acid functionalized nanocarbon $(5 \mathrm{mg})$ was taken in DMF $(20 \mathrm{~mL})$, sonicated for $10 \mathrm{~min}$ to get a homogenous mixture. To this mixture DCC (1 mg) and monoprotected boc-1,2-ethylenediamine $(1 \mathrm{~mL})$ were added. The reaction mixture was stirred for $4 \mathrm{~h}$ under inert atmosphere and the products were collected by centrifugation. The product was washed with copious amounts of water, acetonitrile and ethanol, dried in vacuo. The mono boc-1,2-ethylenediamine modified nanocarbon was subjected to boc-deprotection using 50\% trifluoroacetic acid in dichloromethane for $1 \mathrm{~h}$. The EDnanocarbon containing free amine was dried in vacuo and used for further reaction. The ED functionalized nanocarbon $(5 \mathrm{mg})$ and an acid functionalized nanocarbon $(5 \mathrm{mg})$ were dispersed in DMF $(20 \mathrm{~mL})$ and sonicated for $10 \mathrm{~min}$ for thorough mixing. To this mixture DCC (1 mg) was added and reaction was stirred for $4 \mathrm{~h}$ under inert atmosphere and the conjugate was collected by centrifugation. The product was washed with water, acetonitrile and ethanol, dried in vacuo and used for characterization.
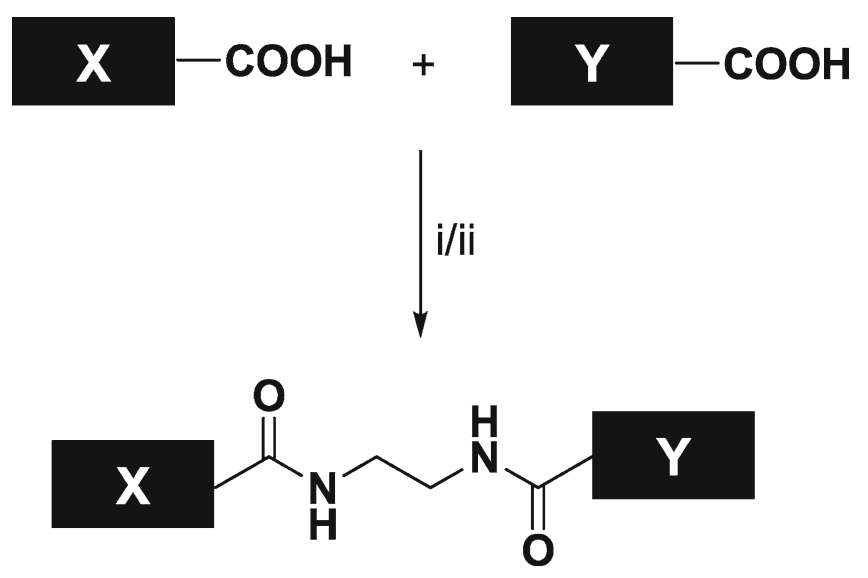

Scheme 1. Preparation of binary conjugates. Nanocarbons $(\mathbf{X}, \mathbf{Y})$ : ND, SWNT and $\mathrm{G}$ containing $\mathrm{COOH}$ groups. (i) For homoconjugates: nanocarbon $(\mathbf{X}=\mathbf{Y})$, DCC, ED, DMF, $4 \mathrm{~h}$. (ii) For heteroconjugates: (a) nanocarbon $(\mathbf{X})$, mono-bocethylenediamine, DCC, DMF, $4 \mathrm{~h}$ followed by TFA-DCM $(1: 1), 1 \mathrm{~h}$. and (b) nanocarbon $(\mathbf{Y}, \mathbf{Y} \neq \mathbf{X}), \mathrm{DCC}, \mathrm{DMF}$, $4 \mathrm{~h}$. Homo- and hetero-conjugates prepared: ND-ND (1), G-G (2), SWNT-SWNT (3), ND-G (4), G-SWNT (5) and ND-SWNT (6). 


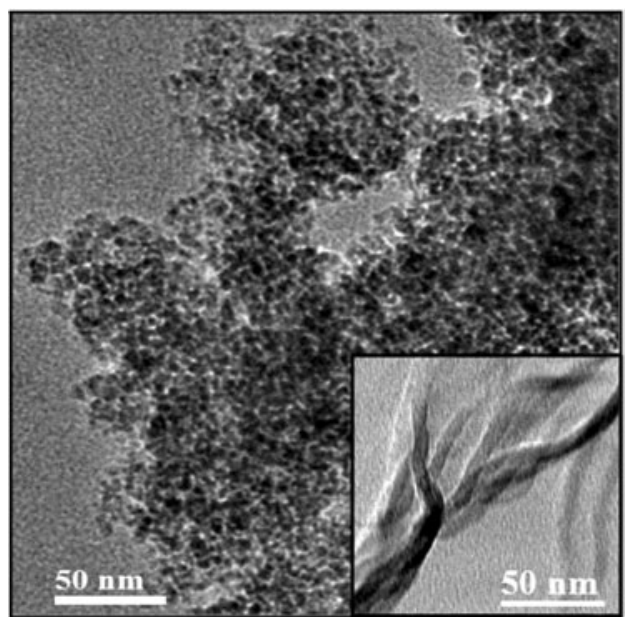

(a)

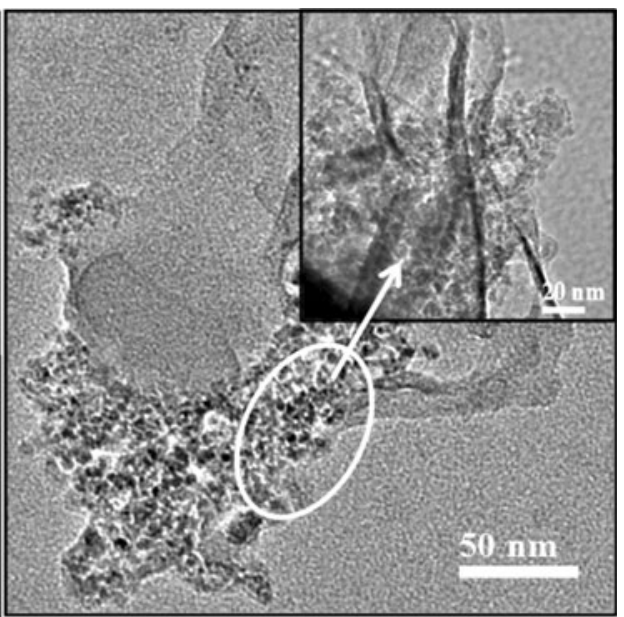

(b)

Figure 1. TEM images of (a) ND-ND conjugate and a G-G conjugate (in the inset) and (b) of a ND-G conjugate (with magnified version in the inset).

The homo- and hetero-conjugates of nanocarbons were characterized using spectroscopic and microscopic techniques. Transmission electron microscope (TEM) micrographs were obtained with a JEOL JEM 3010 , operating with an accelerating voltage of $300 \mathrm{kV}$. Infrared (IR) spectra were recorded on Bruker IFS 66v/S spectrometer. Raman spectra were recorded with a LabRAM HR high-resolution Raman spectrometer (Horiba-Jobin Yvon) using a He-Ne laser $(\mathrm{k}=632.8)$. Photoluminescence (PL) spectra were recorded with a Perkin-Elmer model LS55 luminescence spectrometer.

\section{Results and discussion}

Homo- and hetero-conjugates of ND, SWNT and G were prepared as described in scheme 1 by using 1,2- ethylenediamine linker to selectively conjugate two same or different nanocarbons. Nanocarbons of same type were covalently linked through amide linkages to obtain symmetrical homoconjugates in a one-step process, whereas a two-step amide linkage process was followed to obtain heteroconjugates containing two nanocarbons of different dimensionalities. Figure 1a shows the TEM micrographs of ND-ND (1) with G$G$ (2) homoconjugates. ND exists in the form of small clusters and these clusters crosslink to each other as a result of covalent conjugation forming larger clusters. Similarly, graphene sheets crosslink to each other mostly through the edges. In case of ND-G, clusters of ND are covalently crosslinked to G sheets as shown in the image in figure $1 b$. Figures $2 a$ and $b$ show the TEM images of G-SWNT and ND-SWNT heteroconjugates,

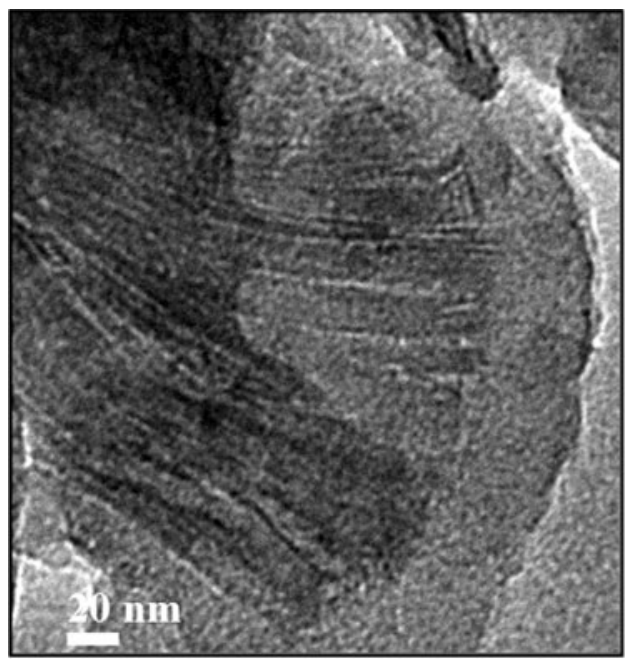

(a)

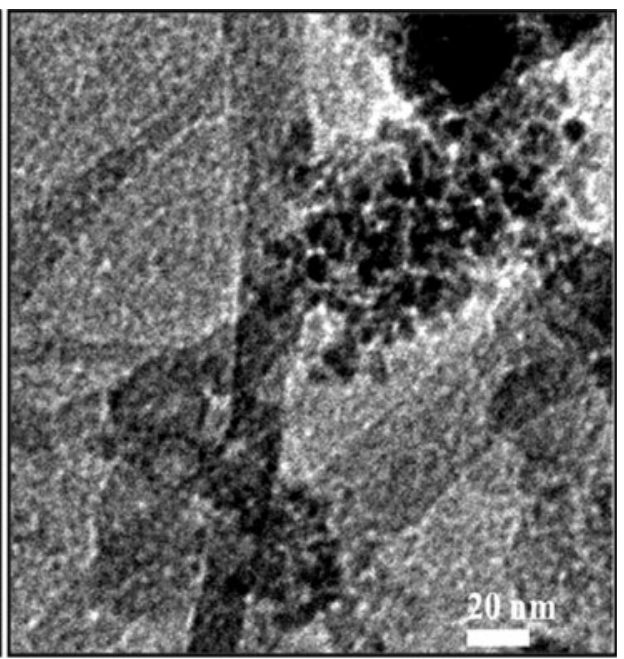

(b)

Figure 2. TEM image of (a) G-SWNT conjugate and (b) ND-SWNT conjugate. 
respectively. The association of two nanocarbons could be observed uniformly throughout the sample due to the crosslinking of the SWNT bundles with $G$ sheets and with the ND clusters. Definitive evidence for covalent crosslinking between nanocarbons to form homoor hetero-conjugates is provided by spectroscopic studies. Thus, infrared spectroscopy clearly shows characteristic bands arising from conjugating as can be seen from typical spectra shown in figure 3 , in the case of homo- and hetero-conjugates formed by graphene.

We see the presence of amide bands in the spectra of conjugates besides $\mathrm{C}-\mathrm{H}$ stretching band arising from the linker. In the covalent binary conjugates the $\mathrm{C}=\mathrm{O}$ peak shifted to lower frequencies $\left(1715 \mathrm{~cm}^{-1}\right)$ than that of corresponding acids $\left(1730 \mathrm{~cm}^{-1}\right)$.

Raman spectroscopy also provides evidence for crosslinking especially in the case of heteroconjugates. We have shown Raman spectra of the conjugates formed by graphene and to illustrate this aspect. We see that G-ND shows the $2 \mathrm{D}$ band $\left(\sim 2600 \mathrm{~cm}^{-1}\right)$ characteristic of the few-layer grapheme, ${ }^{23}$ the band being absent in the spectrum of ND (figure 4). The Raman spectrum of SWNT-G shows characteristic bands due to the

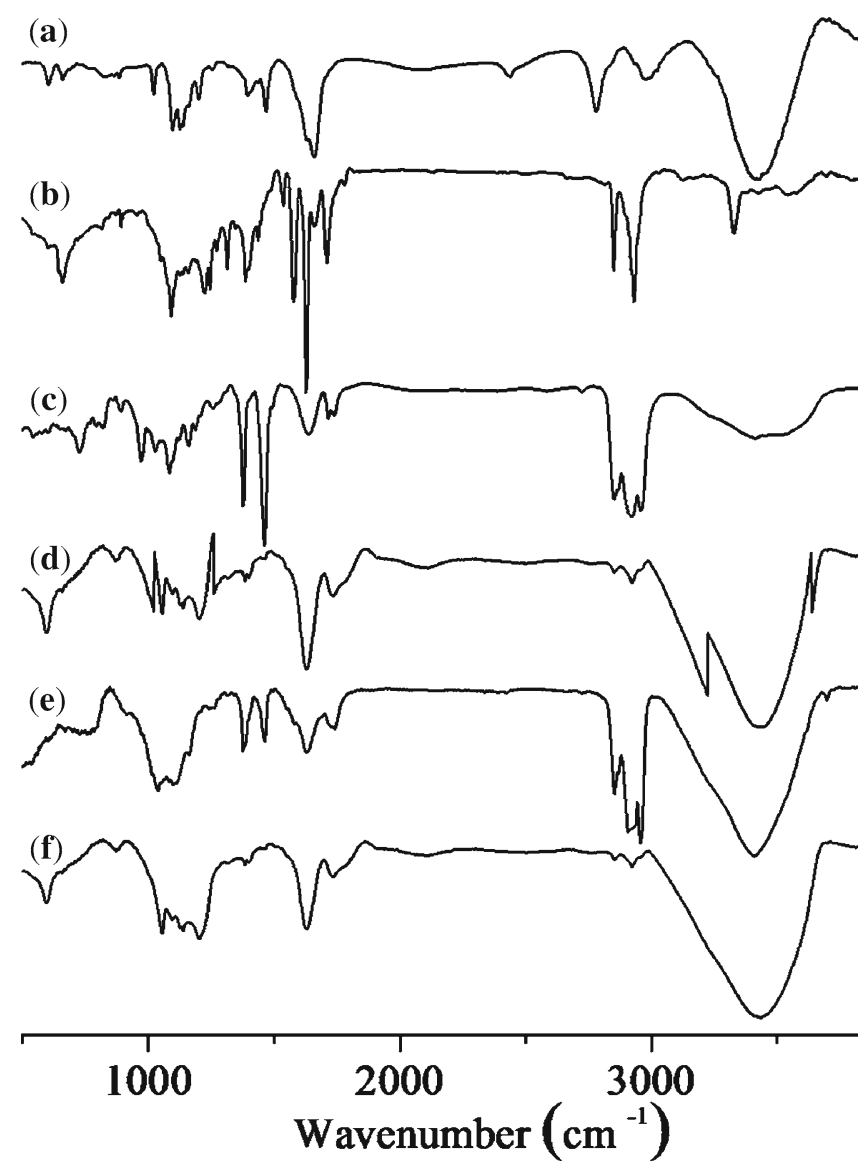

Figure 3. IR spectra of (a) ND-SWNT, (b) ND-G, (c) ND$\mathrm{ND},(\mathbf{d}) \mathrm{SWNT}-\mathrm{COOH},(\mathbf{e}) \mathrm{G}-\mathrm{COOH}$ and (f) ND-COOH.

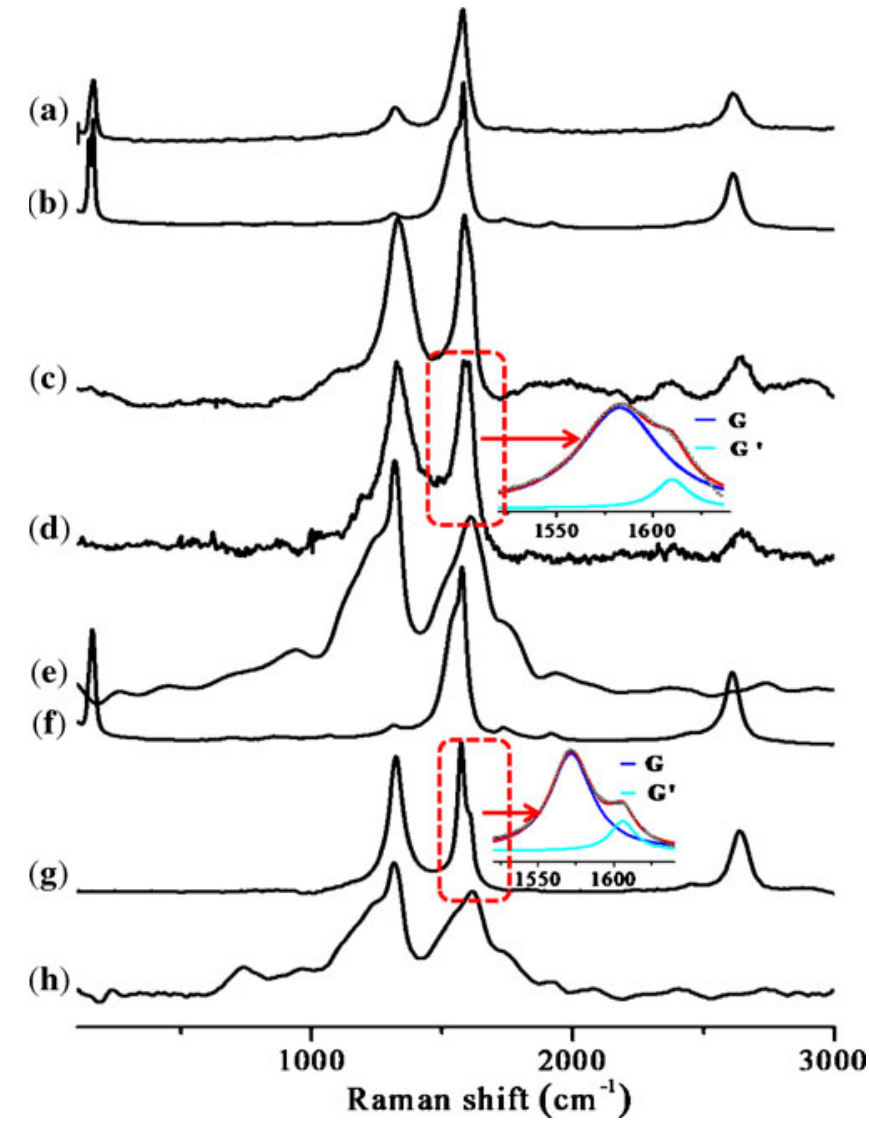

Figure 4. Raman spectra of (a) G-SWNT, (b) ND-SWNT, (c) ND-G, (d) G-G, (e) ND-ND, (f) SWNT-COOH, (g) G$\mathrm{COOH}$ and (h) ND-COOH.

radial breathing mode $(\mathrm{RBM})$ of the SWNTs $(\sim 140$ and $\left.160 \mathrm{~cm}^{-1}\right){ }^{24}$ These bands are also seen in the spectrum of SWNT-ND. The ratio of the intensities of the 2D and

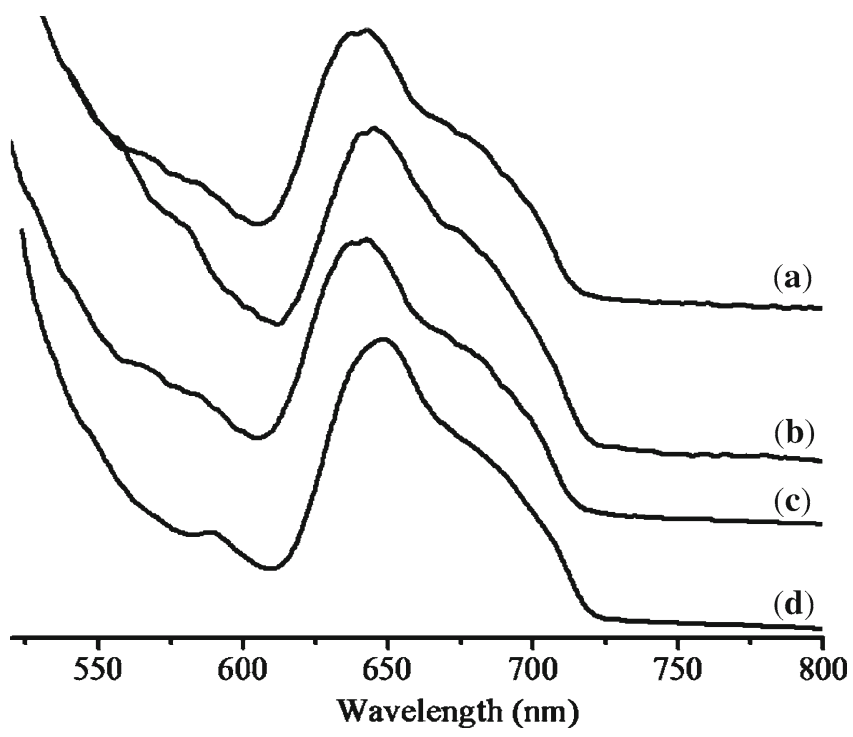

Figure 5. Photoluminescence spectra of (a) ND-SWNT, (b) ND-G, (c) ND-ND and (d) ND-COOH. 


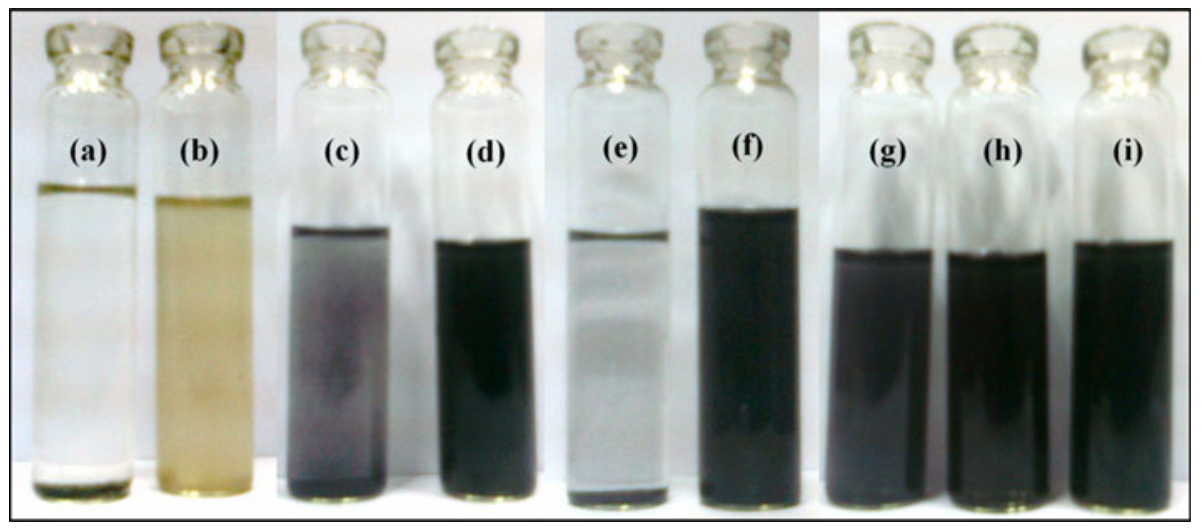

Figure 6. Photographs of dispersions of (a) ND-COOH, (b) ND-ND, (c) G-COOH, (d) G-G, (e) SWNT-COOH, (f) SWNT-SWNT, (g) ND-SWNT, (h) ND-G and (i) GSWNT in DMF.

G Raman bands, $\mathrm{I}_{2 \mathrm{D}} / \mathrm{I}_{\mathrm{G}}$ in graphene conjugate is smaller than in the graphene showing the effect of crosslinking of the electronic structure of graphene. It may be noted that the $\mathrm{I}_{2 \mathrm{D}} / \mathrm{I}_{\mathrm{G}}$ ratio is very sensitive to doping and with electron donating and withdrawing molecules reduce the $\mathrm{I}_{2 \mathrm{D}} / \mathrm{I}_{\mathrm{G}}$ ratio of grapheme. ${ }^{25}$

The photoluminescence spectrum of ND $(\sim 650 \mathrm{~nm})$ is seen in all the conjugates containing ND (figure 5). The photoluminescence bands of the binary composites show a red shift as compared to that of ND-COOH. The observed photoluminescence in case of ND and ND containing binary conjugates originates from the nitrogen and vacancies (NV centres) as reported in the literature. ${ }^{26,27}$ Though we have not understood the actual reason for red-shift observed in the photoluminescence of ND containing binary conjugates, it is a clear evidence of synergistic effect transferred from individual carbon nanostructures to their binary conjugates.

Interestingly, all the covalently crosslinked binary conjugates of nanocarbons form stable homogenous dispersions in dimethyl formamide. In figure 6 we show the photographs of dispersion of acid treated nanocarbons and their conjugates in DMF. Acid treated nanocarbons do not disperse well in DMF while all the homo- and hetero-binary conjugates disperse and at least for $6 \mathrm{~h}$ at a concentration of $1 \mathrm{mg} / 3 \mathrm{~mL}$. This better dispersibility of the binary conjugates can be due to the presence of unused amine groups left on the surface of nanocarbons in binary conjugates.

The dispersions of nanocarbon conjugates can be used for the preparation of polymer-nanofiller composites by using polymers that dissolve in DMF. Thus, we were able to prepare homogenous films of polyethylene oxide (PEO, molecular weight 20,000 g) containing binary nanocarbon composite fillers. Mechanical properties of homo- and hetero-binary conjugates of carbon nanostructures and their polymer composites can be studied to explore the applications related to high performance materials.

\section{Conclusions}

In conclusion, we have successfully prepared covalent homo- and hetero-binary conjugates of nanodiamond, SWNT and graphene, by using a diamine as the crosslinking agent. This procedure is convenient and effective for conjugation of two nanocarbon moieties through amide linkages. The binary conjugates of nanocarbons form stable dispersions in dimethyl formamide. These dispersions of nanocarbon binary conjugates can be used to prepare composites with polymers. The homo- and hetero-binary conjugates of carbon nanostructures and their polymer composites may find applications related to high performance materials.

\section{Supporting information}

Supporting information figure S1 (Raman spectral data), figures S2-S3 (NMR and mass data), table S1 (Raman spectral data), can be seen in www.ias.ac. in/chemsci.

\section{Acknowledgements}

Authors thank Prof. C N R Rao, Jawaharlal Nehru Centre for Advanced Scientific Research for constant support and encouragement.

\section{References}

1. D’Evelyn M P 1998 Surface Properties of diamond. In Handbook of industrial diamonds and diamond films (eds.) M A Prelas, G Popovici and L K Bigelow (New York: Marcel Dekker Inc.) p 89 
2. Dolmatov V Y 2001 Russ. Chem. Rev. 70607

3. Zhu W, Kochanski G P and Jin S 1998 Science 282 1471

4. Yu S J, Kang M W, Chang H C, Chen K M and Yu Y C 2005 J. Am. Chem. Soc. 12717604

5. Fu C C, Lee H Y, Chen K, Lim T S, Wu H Y, Lin P K, Wei P K, Tsao P H, Chang H C and Fann W 2007 Proc. Natl. Acad. Sci. USA 104727

6. Zhao W, Qiu J X Q and Chen H Y 2006 Biosens. Bioelectron. 22649

7. Rao C N R and Govindaraj A 2005 Nanotubes and Nanowires; Royal Society of Chemistry: Cambridge, UK

8. Saito R, Dresselhaus G and Dresselhaus M S 1998 Physical Properties of Carbon Nanotubes, Imperial College Press: London, UK

9. (a) Dai H 2002 Acc. Chem. Res. 35 1035; (b) Banerjee S, Hemraj-Benny T and Wong S-S 2005 Adv. Mater. 17 17

10. Rao C N R, Sood A K, Subrahmanyam K S and Govindaraj A 2009 Angew. Chem. Int. Ed. 487752

11. Prasad K E, Das B, Maitra U, Ramamurty U and Rao C N R 2009 Proc. Natl. Acad. Sci. USA 10613186

12. Guglielmotti V, Chieppa S, Orlanducci S, Tamburri E, Toschi F, Terranova M L and Rossi M 2009 Appl. Phys. Lett. 95222113

13. Chiu P W, Duesburg G S, Wegiikowska U D and Roth $\mathrm{S}$ 2002 Appl. Phys. Lett. 803811

14. Frehill F, Vos J G, Benrezzak S, Koós A A, Kónya Z, Rüther M G, Blau W J, Fonseca A, Nagy J B, Biró L P,
Minett A I and Panhuis M 2002 J. Am. Chem. Soc. 124 13694

15. Holzinger M, Steinmetz J, Samaille D, Glerup M, Paillet M, Bernier P, Ley L and Graupner R 2004 Carbon 42 941

16. Avinash M B, Subrahmanyam K S, Sundarayya Y and Govindaraju T 2010 Nanoscale 21762

17. Dresselhaus M S, Jorio A and Saito R 2010 Annu. Rev. Condens. Matter Phys. 189

18. Dresselhaus M S and Eklund P C 2000 Adv. Phys. 49 705

19. Das B, Voggu R, Rout C S and Rao C N R 2008 Chem. Commun. 415155

20. Weglikowska U D, Benoit J M, Chiu P W, Graupner R, Lebedkin S and Roth S 2002 Curr. Appl. Phys. 2497

21. Rao C N R, Biswas K, Subrahmanyam K S and Govindaraj A 2009 J. Mater. Chem. 192457

22. Jaramillo D, Wheate N J, Ralph S F, Howard W A, Tor Y, Aldrich-Wright J R 2006 Inorg. Chem. 456004

23. Maitra U, Gomathi A and Rao C N R 2008 J. Exp. Nanosci. 3271

24. Subrahmanyam K S, Ghosh A, Gomathi A, Govindaraj A and Rao C N R 2009 Nanosci. Nanotech. Lett. 128

25. Rao C N R, Sood A K, Voggu R, Subrahmanyam K S 2010 J. Phys. Chem. Lett. 1572

26. Chung P-H, Perevedentseva E and Cheng C-L 2007 Surf. Sci. 6013866

27. Vlasov I I, Shenderova O, Turner S, Lebedev O I, Basov A A, Sildos I, Rähn M, Shiryaev A A and Tendeloo G V 2010 Small 6687 